Keywords: giardiasis; infectious diseases; travelers' diarrhea; undergraduate education and training; corrigendum

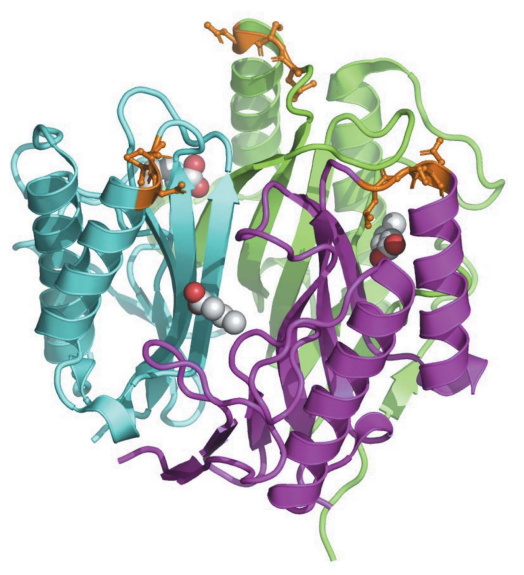

OPEN $\odot$ ACCESS

\section{Crystal structure of a hypothetical protein from Giardia lamblia. Corrigendum}

Dylan K. Beard, ${ }^{a}$ Seonna Bristol, ${ }^{a}$ Kayla Cosby, ${ }^{a}$ Amber Davis, ${ }^{a}$ Courtney Manning, ${ }^{a}$ Lionel Perry, ${ }^{a}$ Lauren Snapp, ${ }^{a}$ Arian Toy, ${ }^{a}$ Kayla Wheeler, ${ }^{a}$

Jeremy Young, ${ }^{\text {a }}$ Bart Staker, ${ }^{\text {b,c }}$ Tracy L. Arakaki, ${ }^{d}$ Jan Abendroth, ${ }^{\text {b }}$ Sandhya

Subramanian, ${ }^{\mathrm{b}, \mathrm{c}}$ Thomas E. Edwards, ${ }^{\mathrm{b}, \mathrm{c}}$ Peter J. Myler ${ }^{\mathrm{c}}$ and Oluwatoyin A. Asojo ${ }^{\mathrm{a} *}$

a Department of Chemistry and Biochemistry, Hampton University, 100 William R. Harvey Way, Hampton, VA 23668, USA, 'beattle Structural Genomics Center for Infectious Disease (SSGCID), Seattle, Washington, USA, ${ }^{\mathbf{c}}$ Center for Infectious Disease Research, formerly Seattle Biomedical Research Institute, 307 Westlake Avenue North Suite 500,

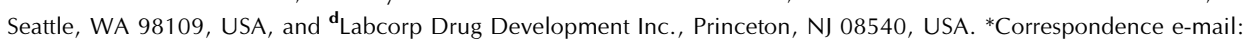
oluwatoyin.asojo@hamptonu.edu

The name of one of the authors in Beard et al. [(2022), Acta Cryst. F78, 59-65] is corrected.

In the article by Beard et al. (2022), the name of one of the authors was given incorrectly. The correct spelling is Sandhya Subramanian.

\section{References}

Beard, D. K., Bristol, S., Cosby, K., Davis, A., Manning, C., Perry, L., Snapp, L., Toy, A., Wheeler, K., Young, J., Staker, B., Arakaki, T. L., Abendroth, J., Subrahamanian, S., Edwards, T. E., Myler, P. J. \& Asojo, O. A. (2022). Acta Cryst. F78, 59-65. 
STRUCTURAL BIOLOGY COMMUNICATIONS

ISSN 2053-230X

Received 13 October 202

Accepted 23 December 2021

Edited by F. T. Tsai, Baylor College of Medicine, Houston, USA

₹ These Hampton University students should be considered co-first authors; their names are listed alphabetically.

Keywords: giardiasis; SSGCID; infectious diseases; travelers' diarrhea; undergraduate education and training; structural genomics.

PDB reference: hypothetical protein from Giardia lamblia, $3 \mathrm{i} 3 \mathrm{f}$

Supporting information: this article has supporting information at journals.iucr.org/f
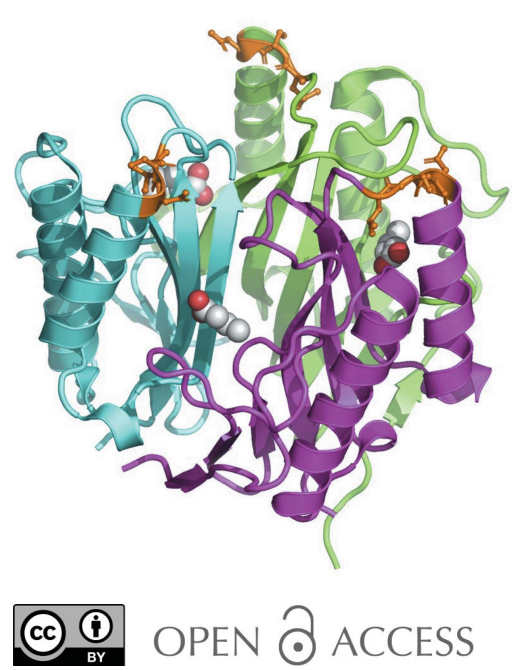

\section{Crystal structure of a hypothetical protein from Giardia lamblia}

Dylan K. Beard, ${ }^{\mathrm{a}} \neq$ Seonna Bristol, ${ }^{\mathrm{a}} \neq$ Kayla Cosby, ${ }^{\mathrm{a}} \neq$ Amber Davis, ${ }^{\mathrm{a}} \neq$ Courtney Manning, ${ }^{a} \ddagger$ Lionel Perry, ${ }^{a} \neq$ Lauren Snapp, ${ }^{a} \neq$ Arian Toy, ${ }^{a} \neq$ Kayla Wheeler, ${ }^{a} \ddagger$ Jeremy Young, ${ }^{a} \ddagger$ Bart Staker,,${ }^{b, c}$ Tracy L. Arakaki, d Jan Abendroth, ${ }^{b}$ Sandhya Subrahamanian, ${ }^{\text {b,c }}$ Thomas E. Edwards, ${ }^{\text {b,c }}$ Peter J. Myler ${ }^{c}$ and Oluwatoyin A. Asojo $^{\mathrm{a} *}$

a Department of Chemistry and Biochemistry, Hampton University, 100 William R. Harvey Way, Hampton, VA 23668, USA, ${ }^{\mathbf{b}}$ Seattle Structural Genomics Center for Infectious Disease (SSGCID), Seattle, Washington, USA, ${ }^{\mathbf{c}}$ Center for Infectious Disease Research, formerly Seattle Biomedical Research Institute, 307 Westlake Avenue North Suite 500, Seattle, WA 98109, USA, and ${ }^{\mathbf{d}}$ Labcorp Drug Development Inc., Princeton, NJ 08540, USA. *Correspondence e-mail: oluwatoyin.asojo@hamptonu.edu

Giardiasis is the most prevalent diarrheal disease globally and affects humans and animals. It is a significant problem in developing countries, the number one cause of travelers' diarrhea and affects children and immunocompromised individuals, especially HIV-infected individuals. Giardiasis is treated with antibiotics (tinidazole and metronidazole) that are also used for other infections such as trichomoniasis. The ongoing search for new therapeutics for giardiasis includes characterizing the structure and function of proteins from the causative protozoan Giardia lamblia. These proteins include hypothetical proteins that share $30 \%$ sequence identity or less with proteins of known structure. Here, the atomic resolution structure of a $15.6 \mathrm{kDa}$ protein was determined by molecular replacement. The structure has the two-layer $\alpha \beta$-sandwich topology observed in the prototypical endoribonucleases L-PSPs (liver perchloric acid-soluble proteins) with conserved allosteric active sites containing small molecules from the crystallization solution. This article is an educational collaboration between Hampton University and the Seattle Structural Genomics Center for Infectious Disease.

\section{Introduction}

The flagellated protozoa Giardia lamblia is the most commonly identified intestinal parasite globally, causing giardiasis, otherwise known as travelers' diarrhea (Daniels et al., 2015; Escobedo et al., 2015). Giardiasis is a zoonotic infection, and Giardia species have been isolated from the stools of vertebrates, including mammals, amphibians and birds (Thompson, 2013). Giardia is an endemic neglected tropical disease, and outbreaks of giardiasis from contaminated water or food sources are common in developing countries because of poor sanitation (McIntyre et al., 2014). It only takes $\sim 10$ Giardia cysts to cause infection, and in developed countries giardiasis is more common among children and hospital patients, especially immunocompromised individuals and institutionalized patients (Huang \& White, 2006). The current standard treatment for giardiasis is antibiotic therapy using tinidazole and metronidazole (Lobovská \& Nohýnková, 2003). Characterizing the structures and functions of G. lamblia proteins is the first step towards identifying new therapeutics for giardiasis.

G. lamblia is one of the organisms selected by the Seattle Structural Genomics Center for Infectious Disease (SSGCID) for high-throughput structural studies, and hypothetical 
proteins have been identified with limited sequence similarity to proteins of known function. One of these hypothetical proteins is a 141-amino-acid protein (UniProt ID A8BD71, XP_001707732.1). This protein shares over 30\% sequence identity and $50 \%$ coverage with only two unique proteins in the Protein Data Bank. One of these proteins is a putative endonuclease from Entamoeba histolytica (PDB entries 3mqw, $3 \mathrm{~m} 1 \mathrm{x}$ and $3 \mathrm{~m} 4 \mathrm{~s} ; 36 \%$ sequence identity and $56 \%$ coverage; Seattle Structural Genomics Center for Infectious Disease, unpublished work). The other comprises the amino-terminal residues 13-121 of Saccharomyces cerevisiae mitochondrial matrix protein Mmf1 (PDB entry 3quw), with 30\% sequence identity and $77 \%$ coverage ( $\mathrm{Pu}$ et al., 2011). A BLAST search against all redundant Giardia sequences reveals three proteins that share appreciable sequence similarity with this hypothetical protein: EFO62390.1, the hypothetical protein GLP15_656 from G. lamblia P15, EET01624.1, the hypothetical protein GL50581_1093 from G. intestinalis ATCC 50581, and ESU43034.1, a putative YjgF/YER057c/UK114 family protein from $G$. intestinalis (Fig. 1). Here, we present the atomic resolution crystal structure of this hypothetical protein as a first step towards clarifying its possible functions.

\section{Materials and methods}

\subsection{Macromolecule production}

The protein was cloned, expressed and purified following standard protocols of the Seattle Structural Genomics Center for Infectious Disease (SSGCID; Bryan et al., 2011; Choi et al., 2011; Serbzhinskiy et al., 2015). Briefly, genomic DNA from
Table 1

Macromolecule-production information.

\begin{tabular}{|c|c|}
\hline Source organism & Giardia lamblia GL50803_14299 \\
\hline DNA source & $\begin{array}{l}\text { Genomic DNA from Dr Ethan A. Merritt, } \\
\text { University of Washington }\end{array}$ \\
\hline Forward primer & $\frac{\text { GGGTCCTGGTTCGATGTTGACGGACTATCG }}{\text { CATCCG }}$ \\
\hline Reverse primer & $\frac{\text { CTTGTTCGTGCTGTTTATTATACGAGGATG }}{\text { GTCCAGCAATCG }}$ \\
\hline Cloning vector & pAVA0421 \\
\hline Expression vector & pAVAC \\
\hline Expression host & E. coli BL21(DE3)R3 Rosetta \\
\hline $\begin{array}{l}\text { Complete amino-acid sequence } \\
\text { of the construct produced }\end{array}$ & $\begin{array}{l}\text { MAHHHHHHMGTLEAQTQGPGSMIYGILSKN } \\
\text { LGMPTPTFLVCPDVVKFENVGQIAVVNG } \\
\text { MVYLGGSVGIDKSGTLHKGLEEQTRQTF } \\
\text { DNIRKCLEYANSGLYIVSLNIFLSTSL } \\
\text { SDSEEARFNELYREVFCVPATRPCRCCV } \\
\text { RAQLQEGLLVEVVNVVAAQK }\end{array}$ \\
\hline $\begin{array}{l}\text { Amino-acid sequence after } 3 \mathrm{C} \\
\text { protease cleavage }\end{array}$ & $\begin{array}{l}\text { GPGSMIYGILSKNLGMPTPTFLVCPDVVKF } \\
\text { ENVGQIAVVNGMVYLGGSVGIDKSGTLH } \\
\text { KGLEEQTRQTFDNIRKCLEYANSGLDYI } \\
\text { VSLNIFLSTSLSDSEEARFNELYREVFC } \\
\text { VPATRPCRCCVRAQLQEGLLVEVVNVVA } \\
\text { AQK }\end{array}$ \\
\hline
\end{tabular}

G. lamblia GL50803_14299 was provided by Dr Ethan Merritt, University of Washington. DNA encoding amino acids 1-141 (UniProt A8BD71) of G. lamblia GL50803_14299 was PCRamplified from genomic DNA using the primers shown in Table 1. The PCR product was cloned into expression vector pAVA0421 (Choi et al., 2011) by ligation-independent cloning (LIC; Aslanidis \& de Jong, 1990). The final expression vector includes a cleavable $6 \times$ His fusion tag followed by the human rhinovirus $3 \mathrm{C}$ protease-cleavage sequence (MAHHHHHHM GTLEAQTQGPGS-ORF). The underlined glutamine (Q) and glycine $(\mathrm{G})$ residues denote the $3 \mathrm{C}$ cleavage site. Plasmid
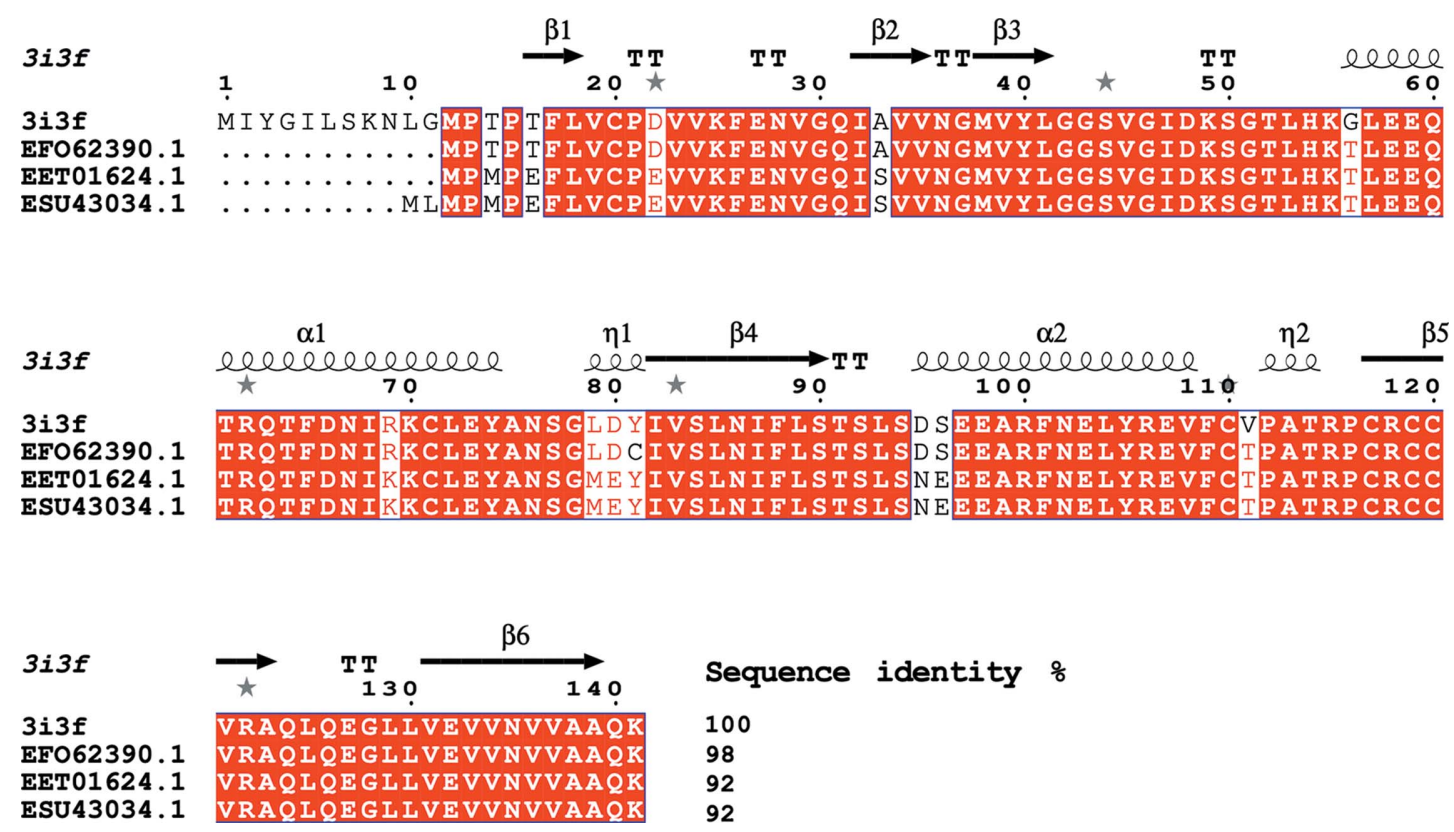

Figure 1

Structural and primary-sequence alignment of the hypothetical protein from G. lamblia (GilaA.00312.a) with EFO62390.1, the hypothetical protein GLP15_656 from G. lamblia P15, EET01624.1, the hypothetical protein GL50581_1093 from G. intestinalis ATCC 50581, and ESU43034.1, a putative YjgF/YER057c/UK114 family protein from $G$. intestinalis. The secondary-structure elements shown are $\alpha$-helices $(\alpha), 3_{10}$-helices $(\eta), \beta$-strands $(\beta)$ and $\beta$-turns (TT). Identical residues are shown in white on a red background and conserved residues are shown in red. This figure was generated using ESPript (Gouet et al., 1999, 2003). 
Table 2

Crystallization.

\begin{tabular}{ll}
\hline Method & Sitting-drop vapor diffusion \\
Plate type & 96 -well Compact 300, Rigaku \\
Temperature $(\mathrm{K})$ & 290 \\
Protein concentration $\left(\mathrm{mg} \mathrm{ml}^{-1}\right)$ & 13.46 \\
Buffer composition of protein & $20 \mathrm{~m} M$ HEPES $\mathrm{pH} 7.0,300 \mathrm{~m} M \mathrm{NaCl}$, \\
$\quad$ solution & $5 \%$ glycerol, $1 \mathrm{~m} M$ TCEP \\
Composition of reservoir solution & $100 \mathrm{~m} M$ Tris $\mathrm{pH} 5.5,25 \%(w / v)$ PEG \\
& $3350,200 \mathrm{~m} M$ ammonium acetate \\
Volume and ratio of drop & $0.4 \mu \mathrm{l}$ protein solution plus $0.4 \mu \mathrm{l}$ \\
& reservoir solution \\
Volume of reservoir $(\mu \mathrm{l})$ & 80 \\
\hline
\end{tabular}

DNA was transformed into chemically competent Escherichia coli BL21(DE3)R3 Rosetta cells. The cells were tested for expression and 21 of culture was grown using auto-induction medium (Studier, 2005) in a LEX Bioreactor (Epiphyte Three Inc.). The expression clone was assigned the SSGCID target identifier GilaA.00312.a.

Both the expression clone and purified protein are available at https://www.ssgcid.org/available-materials/.

The recombinant protein was purified using a four-step protocol consisting of an $\mathrm{Ni}^{2+}$-affinity chromatography (IMAC) step, cleavage of the $\mathrm{N}$-terminal histidine tag with 3C protease, reverse capture with a second $\mathrm{Ni}^{2+}$-affinity chromatography column and size-exclusion chromatography (SEC). All chromatography runs were performed on an ÄKTApurifier 10 (GE) using automated IMAC and SEC programs according to previously described procedures (Bryan et al., 2011). The final SEC was performed on a HiLoad 26/600 Superdex 75 column (GE Healthcare) using a mobile phase consisting of $500 \mathrm{~m} M \mathrm{NaCl}, 25 \mathrm{~m} M$ HEPES, $5 \%$ glycerol, $0.025 \%$ azide, $2 \mathrm{~m} M$ DTT $\mathrm{pH}$ 7.0. Peak fractions were pooled and analyzed using SDS-PAGE. The peak fractions were concentrated to $30.5 \mathrm{mg} \mathrm{ml}^{-1}$ using an Amicon purification system (Millipore). Aliquots of $200 \mu \mathrm{l}$ were flash-frozen in liquid nitrogen and stored at $-80^{\circ} \mathrm{C}$ until use for crystallization.

\subsection{Crystallization}

Crystals were grown following established crystallization approaches at the SSGCID. Briefly, recombinant GilaA.00312.a was diluted to $13.46 \mathrm{mg} \mathrm{ml}^{-1}$. Protein concentration was assessed using the $\mathrm{OD}_{280}$ with a molar extinction coefficient of $7450 \mathrm{M}^{-1} \mathrm{~cm}^{-1}$. Single crystals were obtained by vapor diffusion in sitting drops using equal volumes of protein solution and precipitant solution equilibrated against a reservoir containing precipitant solution (Table 2).

\subsection{Data collection and processing}

Data collection and processing were performed using established protocols at the SSGCID. Specifically, a single crystal was transferred into cryosolution (buffer solution plus $20 \%$ ethylene glycol), flash-cooled in liquid nitrogen and transferred into a puck for data collection on APS beamline 21-ID-F. Data were processed using XDS/XSCALE (Kabsch, 2010). Additional data-collection information is provided in Table 3.
Table 3

Data collection and processing.

Values in parentheses are for the outer shell.

\begin{tabular}{ll}
\hline Diffraction source & SSRL beamline BL12-2 \\
Wavelength $(\AA)$ & 0.9795 \\
Temperature $(\mathrm{K})$ & 100 \\
Detector & ADSC Quantum 315R CCD \\
Space group & $I 4_{1} 22$ \\
$a, b, c(\AA)$ & $119.90,119.90,104.59$ \\
$\alpha, \beta, \gamma\left({ }^{\circ}\right)$ & $90,90,90$ \\
Resolution range $(\AA)$ & $39.41-1.35(1.42-1.35)$ \\
No. of unique reflections & $82643(5692)$ \\
Completeness $(\%)$ & $99.50(96.60)$ \\
Multiplicity & $6.30(4.80)$ \\
$\langle I / \sigma(I)\rangle$ & 13.50 \\
$R_{\text {r.i.m. }}^{\dagger}$ & $0.081(5.40)$ \\
Overall $B$ factor from Wilson plot $\left(\AA^{2}\right)$ & 14.0 \\
\hline
\end{tabular}

$\dagger$ Estimated $R_{\text {r.i.m. }}=R_{\text {merge }}[N /(N-1)]^{1 / 2}$, where $N$ is the data multiplicity.

Table 4

Structure solution and refinement.

Values in parentheses are for the outer shell.

\begin{tabular}{ll}
\hline Resolution range $(\AA)$ & $37.35-1.35(1.38-1.35)$ \\
Completeness $(\%)$ & 99.6 \\
No. of reflections, working set & $82626(5432)$ \\
No. of reflections, test set & $4134(260)$ \\
Final $R_{\text {cryst }}$ & $0.176(0.255)$ \\
Final $R_{\text {free }}$ & $0.191(0.264)$ \\
Cruickshank DPI & 0.056 \\
No. of non-H atoms & \\
$\quad$ Protein & 2971 \\
$\quad$ Ions & 0 \\
Ligand & 20 \\
$\quad$ Water & 443 \\
$\quad$ Total & 3434 \\
R.m.s. deviations & \\
$\quad$ Bonds $(\AA)$ & 0.004 \\
Angles $\left({ }^{\circ}\right)$ & 0.893 \\
Average $B$ factors $\left(\AA^{2}\right)$ & \\
$\quad$ Protein & 13.5 \\
Ligands & 18.5 \\
$\quad$ Water & 23.7 \\
Ramachandran plot & \\
$\quad$ Most favored $(\%)$ & 97.81 \\
Allowed $(\%)$ & 2.19 \\
Outlier $(\%)$ & 0 \\
\hline
\end{tabular}

\subsection{Structure solution and refinement}

The structure was solved by molecular replacement using MOLREP (Lebedev et al., 2008; Vagin \& Teplyakov, 2010) with the structure of yeast mitochondrial matrix factor 1 (PDB entry 1jd1; Deaconescu et al., 2002) as the search model. Initial refinement was carried out with REFMAC (Murshudov et al., 2011) with TLS, with manual refinement in Coot (Emsley \& Cowtan, 2004; Emsley et al., 2010). The structure quality was checked by MolProbity (Headd et al., 2009) and the resulting structure-refinement data are provided in Table 4.

\section{Results and discussion}

Each monomer of the hypothetical G. lamblia protein (GilaA.00312.a; PDB entry 3i3f) folds as a two-layer $\alpha \beta$ sandwich. The quaternary structure is a homotrimer stabilized by seven hydrogen bonds and 99 nonbonded contacts per monomer. The trimer forms a $\beta$-barrel with core $\beta$-sheets 


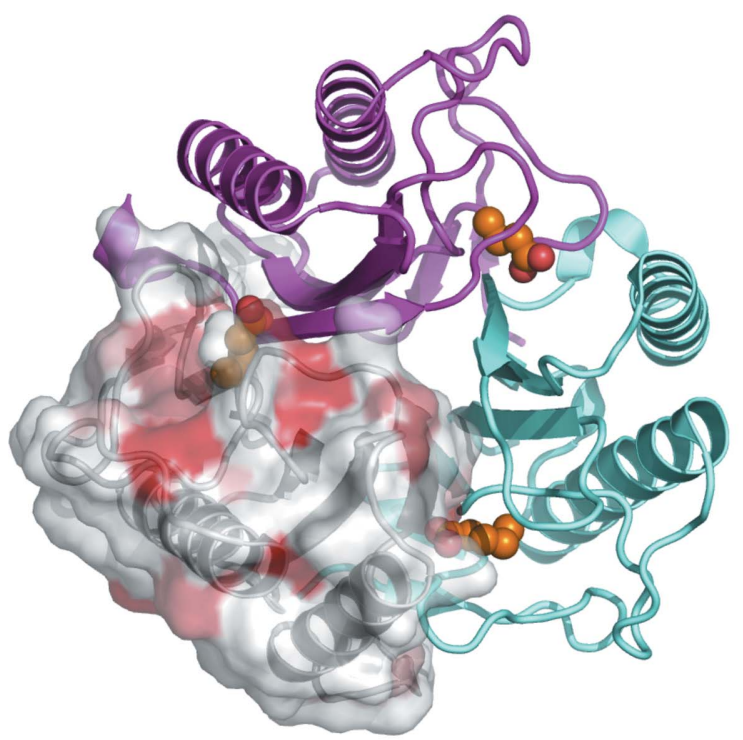

(a)

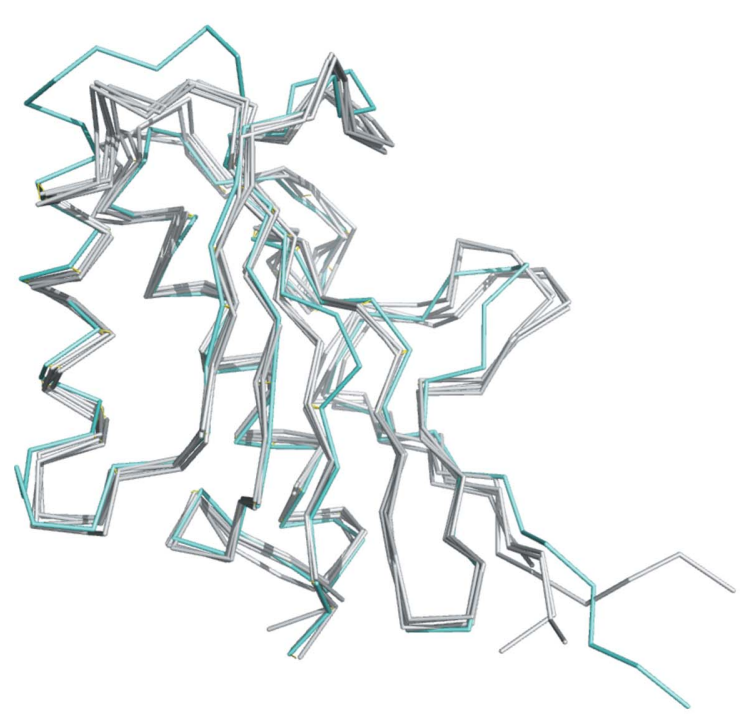

$(c)$

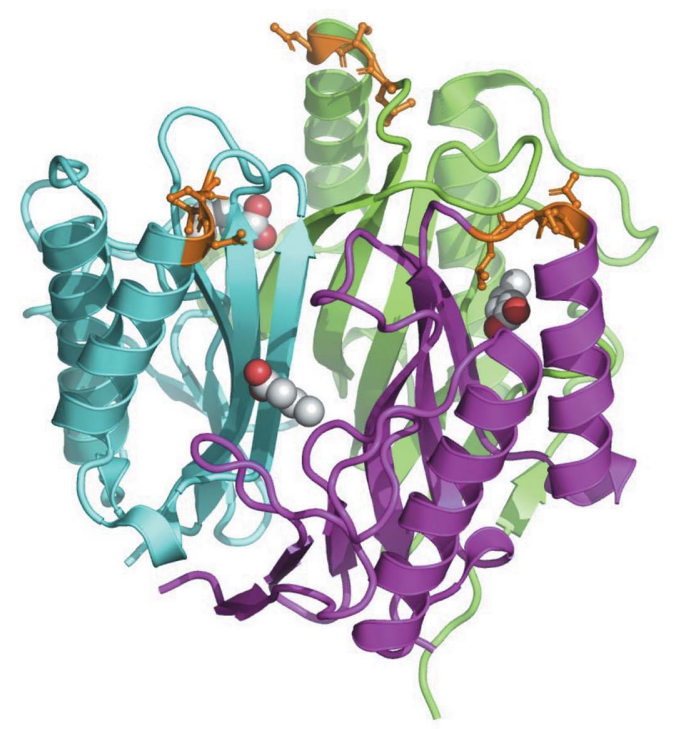

(b)

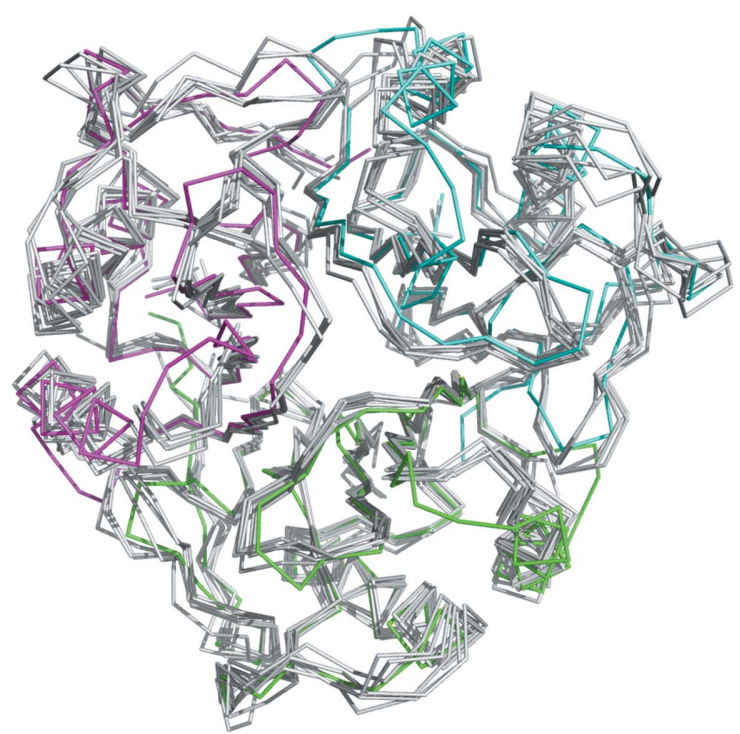

(d)
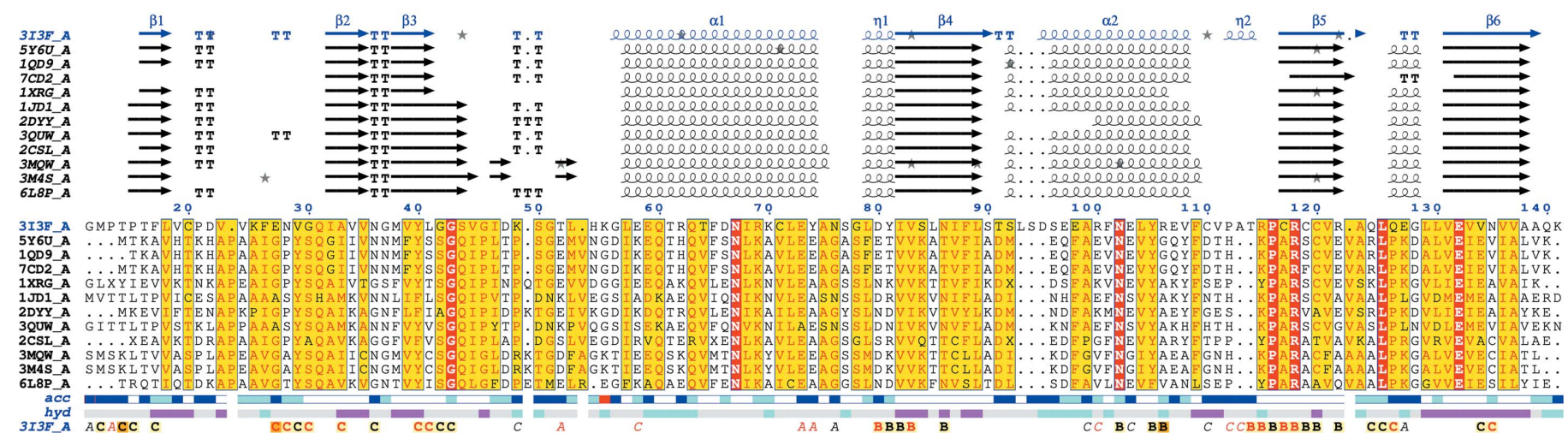

$(e)$

Figure 2

Structure of GilaA.00312.a (PDB entry 3i3f). (a) A GilaA.00312.a trimer with one monomer shown as a white surface; the identical residues in similar proteins are shown in red. (b) Alternative view of the trimer showing the unique insertion found in GilaA.00312.a as gold sticks. Endoribonuclease allosteric sites are identified by the modeled ligands in ball-and-stick representation. (c) Superposition of the GilaA.00312.a monomer (aquamarine) on the closest structures (gray). (d) A GilaA.00312.a trimer (aquamarine) superposed on the closest structures (gray). (e) ENDScript alignment of the closest structures. The secondary-structure elements shown are $\alpha$-helices $(\alpha), 3_{10}$-helices $(\eta), \beta$-strands $(\beta)$ and $\beta$-turns (TT). Identical residues are shown in red and similar residues in yellow. The same structures are used in $(c),(d)$ and $(e)$. 
surrounded by $\alpha$-helices (Fig. 2a). The largest interface between the monomers contains electron density for small molecules, which we built as two molecules of pentanoic acid and one of butanoic acid. The ligands have sufficient electron density, as indicated by composite omit maps (Supplementary Fig. S1), and their $B$ factors are consistent with the contacting protein atoms. It is plausible that these ligands have dual conformations or could have been built with other small molecules (Supplementary Figs. S1 and S2). Nonetheless, the significance of these molecules is that they sit in the largest clefts in the structure. These largest clefts have volumes of $\sim 1850 \AA^{3}$ and are consistent with the allosteric sites observed in other endoribonucleases.

ENDScript (Gouet et al., 2003; Robert \& Gouet, 2014) analysis was used to identify the most similar structures to
GilaA.00312.a (Fig. 2). The most similar structures identified from the analysis were those of YabJ from Bacillus subtilis (PDB entries 5y6u, 1qd9 and 7cd2; Sinha et al., 1999; Fujimoto et al., 2021). A conserved hypothetical protein from Clostridium thermocellum Cth-2968 (PDB entry 1xrg) was identified as the next closest structure. Other similar structures include Saccharomyces cerevisiae homologous mitochondrial matrix factor 1 (PDB entry 1jd1; Deaconescu et al., 2002), a putative translation-initiation inhibitor PH0854 from Pyrococcus horikoshii (PDB entry 2dyy), a putative endonuclease from Entamoeba histolyca (PDB entry 3mqw), Saccharomyces cerevisiae mitochondrial matrix protein Mmf1 (PDB entry 3quw; Pu et al., 2011), TTHA0137 from Thermus thermophilus HB8 (PDB entry 2csl) and RidA from the Antarctic bacterium Psychrobacter sp. (PDB entry 618p; Kwon et al., 2020). The
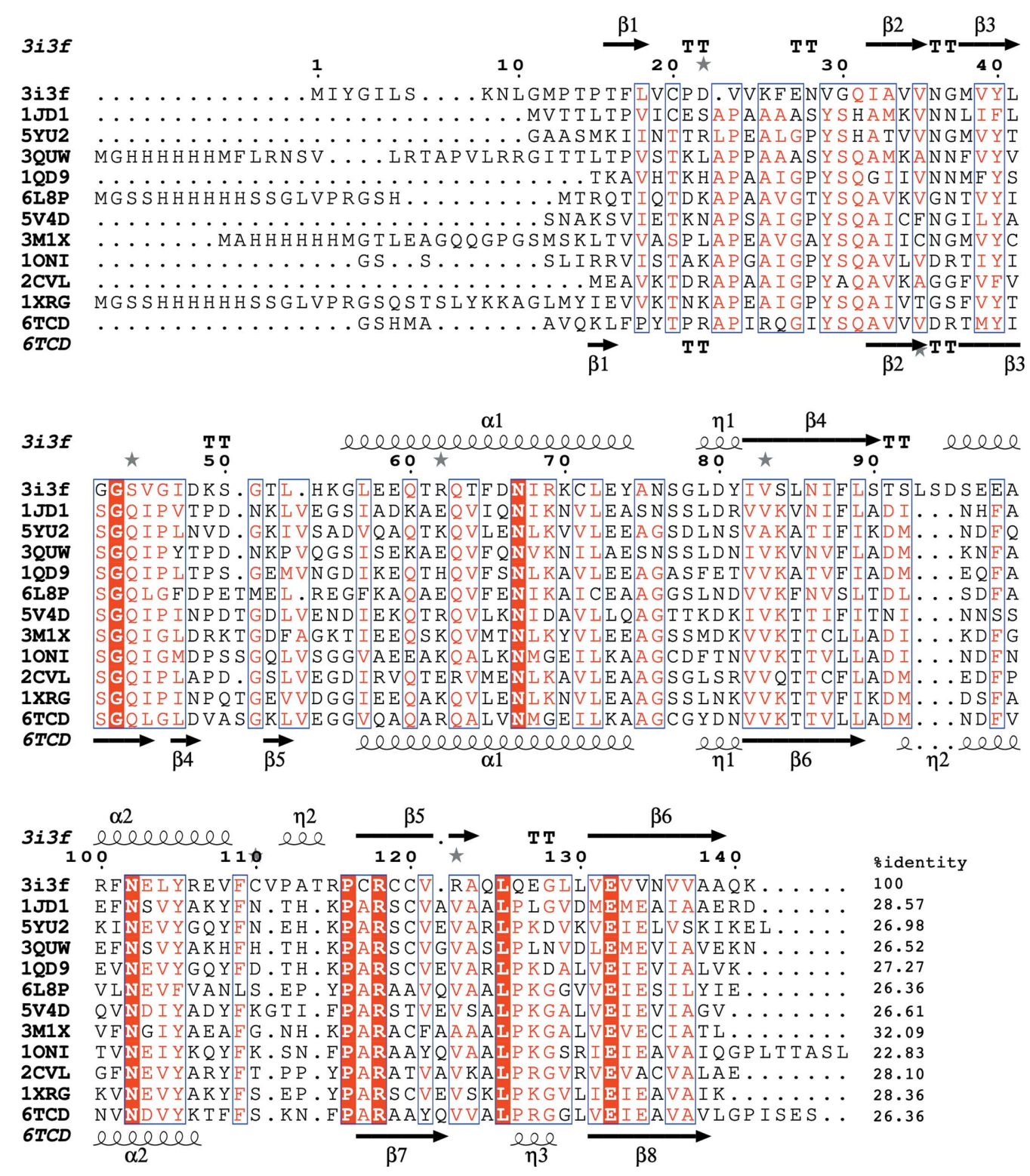

Figure 3

Structural and primary-sequence alignment of GilaA.00312.a and structurally similar YjgF/YER057c/UK114 endoribonucleases. The secondarystructure elements are shown as follows: $\alpha$-helices are shown as large coils, $3_{10}$-helices ae shown as small coils labeled $\eta, \beta$-strands are shown as arrows labeled $\beta$ and $\beta$-turns are labeled TT. Identical residues are shown on a red background; conserved residues are shown in red and conserved regions in blue boxes. This figure was generated using ESPript (Gouet et al., 1999, 2003). 
similar structures identified by ENDScript analysis belong to the YjgF/YER057c/UK114 family of endoribonucleases with L-PSP topology (Kim et al., 2018; Zhang et al., 2010; Volz, 1999). Structural analysis with PDBeFold (http://www.ebi.ac.uk/ msd-srv/ssm/; Krissinel \& Henrick, 2004) and the DALI server (http://ekhidna2.biocenter.helsinki.fi/dali/; Holm, 2020) confirms that GilaA.00312.a has the structural features of the YjgF/YER057c/UK114 family of endoribonucleases (Figs. 2 and 3). Detailed results of the DALI and PDBeFold analysis are included in the supporting information.

The YjgF/YER057c/UK114 family of endoribonucleases belong to the superfamily of proteins known as endoribonuclease L-PSP/chorismate mutase-like (IPR013813). These are homotrimeric proteins in which the intermolecular cavity forms putative allosteric binding sites for small molecules (Mistiniene et al., 2003; Burman et al., 2003, 2007; Miyakawa et al., 2006). The superfamily includes two broadly defined families: YjgF/YER057c/UK114 and AroH chorismate mutases. The YjgF/YER057c/UK114 family are found in bacteria, archaea and eukaryotes (Lambrecht et al., 2012), while AroH chorismate mutases are only found in bacteria. Specific members of the superfamily are YjgF (which was renamed RidA), which is known to deaminate reactive enamine/imine intermediates in pyridoxal $5^{\prime}$-phosphate (PLP)-dependent enzyme reactions (Lambrecht et al., 2012), and the yeast growth inhibitor YER057c, which has roles in the regulation of metabolic pathways and cell differentiation (Kim et al., 2001). Other members include UK114/L-PSP (liver perchloric acid-soluble protein), mammalian translational inhibitor proteins and endoribonucleases that directly affect mRNA translation by inducing disaggregation of the reticulocyte polysomes into $80 \mathrm{~S}$ ribosomes (Morishita et al., 1999). RutC is essential for the ability of $E$. coli to use uracil as a sole nitrogen source and possibly by reducing aminoacrylate peracid to aminoacrylate (Kim et al., 2010), while B. subtilis $\mathrm{YabJ}$ is required for adenine-mediated repression of purinebiosynthetic genes (Sinha et al., 1999). The structural neighbors of GilaA.00312.a are all members of the YjgF/YER057c/ UK114 family and the overall structural topology is well conserved (Figs. 2 and 3). These closest structures share less than 32.1\% sequence with GilaA.00312.a (Fig. 3).

GilaA.00312.a has a unique insertion of three residues (LSD) that differentiates it from its structural neighbors (Fig. 2). These residues follow Ser92 in the loop preceding $\alpha$-helix 2 and form part of the access to the allosteric binding cavity of GilaA.00312.a (Figs. 2 and 3). Furthermore, the allosteric site of GilaA.00312.a differs from the conserved topology of its structural neighbors (Figs. 2 and 3). The significance of this observation will be investigated further since experimental evidence indicate that the metabolic functions of endoribonucleases are mediated by the allosteric site (Niehaus et al., 2015).

\section{Closing remarks}

The structure of a hypothetical protein from G. lamblia (GilaA.00312.a) suggests that it belongs to the $\mathrm{YjgF} /$
YER057c/UK114 family, forming a trimer with allosteric active sites. Future studies are required to determine the ligands that bind to GilaA.00312.a and the specific mechanisms of its functions in the light of the observed unique structural features in its allosteric binding site.

\section{Acknowledgements}

We thank the SSGCID cloning and protein-production groups at the Center for Infectious Disease Research and the University of Washington. This research used resources of the Advanced Photon Source, a US Department of Energy (DOE) Office of Science User Facility operated for the DOE Office of Science by Argonne National Laboratory under Contract No. DE-AC02-06CH11357. Use of LS-CAT Sector 21 was supported by the Michigan Economic Development Corporation and the Michigan Technology Tri-Corridor (Grant 085P1000817).

\section{Funding information}

This work was supported by National Institutes of Health/ National Institute of Allergy and Infectious Diseases (contract Nos. HHSN272201700059C, HHSN272201200025C and HHSN272200700057C to PJM). Hampton University students were part of a Hampton University Chemistry Education and Mentorship Course-based Undergraduate Research pilot (HU-ChEM CURES) funded by the National Institute of General Medical Sciences (award No. 1U01GM138433 to OAA).

\section{References}

Aslanidis, C. \& de Jong, P. J. (1990). Nucleic Acids Res. 18, 6069-6074. Bryan, C. M., Bhandari, J., Napuli, A. J., Leibly, D. J., Choi, R., Kelley, A., Van Voorhis, W. C., Edwards, T. E. \& Stewart, L. J. (2011). Acta Cryst. F67, 1010-1014.

Burman, J. D., Stevenson, C. E. M., Hauton, K. A., Sawers, G. \& Lawson, D. M. (2003). Acta Cryst. D59, 1076-1078.

Burman, J. D., Stevenson, C. E. M., Sawers, R. G. \& Lawson, D. M. (2007). BMC Struct. Biol. 7, 30.

Choi, R., Kelley, A., Leibly, D., Nakazawa Hewitt, S., Napuli, A. \& Van Voorhis, W. (2011). Acta Cryst. F67, 998-1005.

Daniels, M. E., Shrivastava, A., Smith, W. A., Sahu, P., Odagiri, M., Misra, P. R., Panigrahi, P., Suar, M., Clasen, T. \& Jenkins, M. W. (2015). Am. J. Trop. Med. Hyg. 93, 596-600.

Deaconescu, A. M., Roll-Mecak, A., Bonanno, J. B., Gerchman, S. E., Kycia, H., Studier, F. W. \& Burley, S. K. (2002). Proteins, 48, 431436.

Emsley, P. \& Cowtan, K. (2004). Acta Cryst. D60, 2126-2132.

Emsley, P., Lohkamp, B., Scott, W. G. \& Cowtan, K. (2010). Acta Cryst. D66, 486-501.

Escobedo, A. A., Arencibia, R., Vega, R. L., Rodríguez-Morales, A. J., Almirall, P. \& Alfonso, M. (2015). J. Infect. Dev. Ctries, 9, 76-86.

Fujimoto, Z., Hong, L. T. T., Kishine, N., Suzuki, N. \& Kimura, K. (2021). Biosci. Biotechnol. Biochem. 85, 297-306.

Gouet, P., Courcelle, E., Stuart, D. I. \& Métoz, F. (1999). Bioinformatics, 15, 305-308.

Gouet, P., Robert, X. \& Courcelle, E. (2003). Nucleic Acids Res. 31, 3320-3323.

Headd, J. J., Immormino, R. M., Keedy, D. A., Emsley, P., Richardson, D. C. \& Richardson, J. S. (2009). J. Struct. Funct. Genomics, 10, 8393.

Holm, L. (2020). Protein Sci. 29, 128-140. 
Huang, D. B. \& White, A. C. (2006). Gastroenterol. Clin. North Am. 35, 291-314.

Kabsch, W. (2010). Acta Cryst. D66, 125-132.

Kim, H. J., Kwon, A.-R. \& Lee, B.-J. (2018). Biosci. Rep. 38, BSR20180768.

Kim, J.-M., Yoshikawa, H. \& Shirahige, K. (2001). Genes Cells, 6, 507517.

Kim, K.-S., Pelton, J. G., Inwood, W. B., Andersen, U., Kustu, S. \& Wemmer, D. E. (2010). J. Bacteriol. 192, 4089-4102.

Krissinel, E. \& Henrick, K. (2004). Acta Cryst. D60, 2256-2268.

Kwon, S., Lee, C. W., Koh, H. Y., Park, H., Lee, J. H. \& Park, H. H. (2020). Biochem. Biophys. Res. Commun. 522, 585-591.

Lambrecht, J. A., Flynn, J. M. \& Downs, D. M. (2012). J. Biol. Chem. 287, 3454-3461.

Lebedev, A. A., Vagin, A. A. \& Murshudov, G. N. (2008). Acta Cryst. D64, 33-39.

Lobovská, A. \& Nohýnková, E. (2003). Cas. Lek. Cesk. 142, 177-181.

McIntyre, K. M., Setzkorn, C., Wardeh, M., Hepworth, P. J., Radford, A. D. \& Baylis, M. (2014). Prev. Vet. Med. 116, 325-335.

Mistiniene, E., Luksa, V., Sereikaite, J. \& Naktinis, V. (2003). Bioconjug. Chem. 14, 1243-1252.

Miyakawa, T., Lee, W. C., Hatano, K., Kato, Y., Sawano, Y., Miyazono, K., Nagata, K. \& Tanokura, M. (2006). Proteins, 62, 557-561.
Morishita, R., Kawagoshi, A., Sawasaki, T., Madin, K., Ogasawara, T., Oka, T. \& Endo, Y. (1999). J. Biol. Chem. 274, 20688-20692.

Murshudov, G. N., Skubák, P., Lebedev, A. A., Pannu, N. S., Steiner, R. A., Nicholls, R. A., Winn, M. D., Long, F. \& Vagin, A. A. (2011). Acta Cryst. D67, 355-367.

Niehaus, T. D., Gerdes, S., Hodge-Hanson, K., Zhukov, A., Cooper, A. J., ElBadawi-Sidhu, M., Fiehn, O., Downs, D. M. \& Hanson, A. D. (2015). BMC Genomics, 16, 382.

Pu, Y.-G., Jiang, Y.-L., Ye, X.-D., Ma, X.-X., Guo, P.-C., Lian, F.-M., Teng, Y.-B., Chen, Y. \& Zhou, C.-Z. (2011). J. Struct. Biol. 175, 469474.

Robert, X. \& Gouet, P. (2014). Nucleic Acids Res. 42, W320-W324.

Serbzhinskiy, D. A., Clifton, M. C., Sankaran, B., Staker, B. L., Edwards, T. E. \& Myler, P. J. (2015). Acta Cryst. F71, 594-599.

Sinha, S., Rappu, P., Lange, S. C., Mäntsälä, P., Zalkin, H. \& Smith, J. L. (1999). Proc. Natl Acad. Sci. USA, 96, 13074-13079.

Studier, F. W. (2005). Protein Expr. Purif. 41, 207-234.

Thompson, R. C. (2013). Int. J. Parasitol. 43, 1079-1088.

Vagin, A. \& Teplyakov, A. (2010). Acta Cryst. D66, 22-25.

Volz, K. (1999). Protein Sci. 8, 2428-2437.

Zhang, H.-M., Gao, Y., Li, M. \& Chang, W.-R. (2010). Biochem. Biophys. Res. Commun. 397, 82-86. 